\title{
Perspectivas de procesamiento y uso del carbón mineral peruano
}

\author{
Mario Rojas Delgado \\ Ingeniería Industrial n. 26, 2008, ISSN 1025-9929, pp. 231-250
}

Resumen: Este artículo presenta las principales zonas de yacimientos y reservas de carbón mineral en el Perú, los tipos, usos y aplicaciones del carbón mineral; los procesos avanzados basados en tecnologías limpias para la industrialización del carbón mineral y estimados de los costos de producción e inversión en la etapa preliminar de las operaciones para el procesamiento industrial del carbón mineral. También se dan recomendaciones para el desarrollo de la industria del carbón mineral, principalmente en las regiones del norte del país, como La Libertad.

Palabras clave: Carbón mineral, antracita, energía

\section{Perspectives of processing and use of peruvian coal}

Abstract: This paper presents the main zones of coal deposits and reserves in Perú, the types, uses and applications of coal; the advanced processes based in clean technologies for the industrialization of coal, and some estimates of investment and production costs, at preliminary level, for the operations of industrial processing of coal. Several recommendations for developing of a coal industry, mainly in the northern region of the country, remarking the high potential of anthracite in the region La Libertad, are also given.

Keywords: Coal, anthracite, energy 


\section{INTRODUCCIÓN}

La fluctuante y ascendente cotización del petróleo, principal recurso energético usado en el mundo, han generado preocupación en los ámbitos internacional y nacional, lo que está llevando a la búsqueda de combustibles fósiles alternativos, como el carbón mineral, cuyo costo es relativamente competitivo.

En otros países existen plantas demostrativas y comerciales que emplean el carbón mineral en procesos avanzados (aplicaciones emergentes), como las plantas termoeléctricas, la producción de coque para la industria metalúrgica, la producción de un sustituto del gas natural, la generación de energía térmica para la industria metalmecánica y de servicios y la obtención de materiales intermedios para la fabricación de productos químicos.

El Perú alberga un considerable potencial de carbón cuyo aprovechamiento ofrece interesantes y variadas oportunidades para los inversionistas. Las mejores posibilidades se presentan en la industrialización de la antracita existente en los Andes noroccidentales. La reserva potencial de la antracita en la sierra norte es del orden de cientos de millones de toneladas. Este tipo de carbón tiene un alto poder calorífico y puede utilizarse en la industria y en la producción de derivados para la exportación.

La explotación de antracita en el Perú es pequeña y rudimentaria, su transporte es muy caro debido a la falta de infraestructura vial adecuada, la producción es heterogénea y el suministro inestable. La mayor parte de la antracita extraída se utiliza como fuente de energía barata en las ladrilleras, la calefacción comercial y solo una pequeña fracción en las industrias. Por la carencia de un suministro adecuado no se ha desarrollado el mercado para el carbón nacional.

El requisito para el uso racional de la antracita es la preparación, que en condiciones mínimas consistiría en la homogeneización, clasificación por tamaño y lavado. Este trabajo es realizado normalmente por los productores, pero lo pueden hacer también los usuarios e intermediarios. Durante la preparación conviene separar los trozos gruesos, que tienen una mayor cotización. El carbón de mejor calidad se puede 
exportar siempre y cuando se cuente con un lote de tal magnitud que resulte compatible con la capacidad de una embarcación dedicada.

El lavado es especialmente importante para el beneficio de la fracción fina, que constituye una gran parte de la antracita extraída de la mina. Actualmente, esta fracción no se lava y, en consecuencia, el contenido de material no combustible es alto, lo que reduce su cotización. La roca sedimentaria de grano muy fino tiene una temperatura de fusión menor que la de las cenizas del carbón y originan su aglomeración. Esto reduce la temperatura de operación de los hornos de antracita, que produce cenizas en polvo de pequeña magnitud.

El déficit de energía eléctrica en el norte peruano ha sido del orden de cientos de megavatios y continúa aumentando. Para cubrir este déficit se han preparado varios anteproyectos de centrales carboeléctricas basados en las antracitas de las cuencas de los ríos Chicama y Santa, cuyos yacimientos son más accesibles. La energía producida por estas centrales se alimentaría al sistema nacional interconectado, cuya línea de transmisión se extiende a lo largo de toda la costa del norte del Perú. Algunos de estos proyectos alcanzaron el nivel de factibilidad pero no se concretaron por falta de inversión pública y privada (Ingemmet, 1996).

Se plantean las siguientes alternativas para el desarrollo energético e industrial, basado en el aprovechamiento del carbón mineral:

- La gasificación integrada con el empleo de carbón mineral para obtener energía eléctrica, hidrógeno y calor.

- Empleo del carbón mineral en procesos avanzados de combustión para la producción de energía eléctrica.

- Mayor valor agregado al carbón mineral en bruto para conseguir un mejor precio para el carbón procesado.

- Uso del carbón mineral procesado en la industria química, metalmecánica y de servicios.

- Utilización del carbón mineral para la industria metalúrgica, producción de gas sustituto del gas natural y de hidrógeno. 


\section{DISPONIBILIDAD Y RESERVAS DE CARBÓN MINERAL EN EL PERÚ}

En el mapa de reservas carboníferas del Perú (figura 1) se observan las zonas con mayor ocurrencia de yacimientos de carbón tipo antracita, bituminoso y grafito.

Las principales reservas de carbón en el Perú, según el Ministerio de Energía y Minas (Minem, 2003), se ubican en las regiones de La Libertad, Áncash, Lima y Cajamarca. Cabe destacar que las reservas de carbón consignadas son en su mayoría de antracita, carbón butimonoso y subbituminoso.

- La Libertad. En la región La Libertad los principales yacimientos de carbón mineral se ubican en la zona del Alto Chicama. Al año 2003 se registraron 17 unidades mineras con operaciones en Cascas, Marmot, Usquil, Quiruvilca y Poroto. Las reservas en poder de concesionarios registrados varían entre 800 y 410.000 toneladas.

- Áncash. Los principales yacimientos mineros de esta región se ubican en Corongo, Huaylas, Yungay y Bolognesi. Al año 2003 se registraron 33 unidades mineras con operaciones en Bambas, Huallanca, Santa Cruz, Caraz, Pueblo Libre, Nepeña, Yungay, Ranrahirca, San Luis, Mancos y Chavín de Huántar. Las reservas - principalmente de carbón subbituminoso - entregadas a los concesionarios registrados varían entre 20 y 1.300 .000 toneladas.

- Lima. Los principales yacimientos se ubican en Oyón. Al año 2003 se registraron 22 unidades mineras con operaciones en Oyón y Andajes. Las reservas son principalmente de carbón de tipo subbituminoso, antracita y bituminoso. Los concesionarios registrados cuentan con reservas que varían entre 1.000 y 100.000 toneladas.

- Cajamarca. Los principales yacimientos de carbón se ubican en Chota, Hualgayoc, San Marcos, Cajamarca. Al 2003 se registraron seis unidades mineras con operaciones en Chalamarca, Bambamarca, Gregorio Pita, San Juan y Cospán. Los concesionarios registrados tienen reservas que varían entre 500 y 135.000 toneladas. 
Figura 1

Mapa de reservas carboníferas del Perú

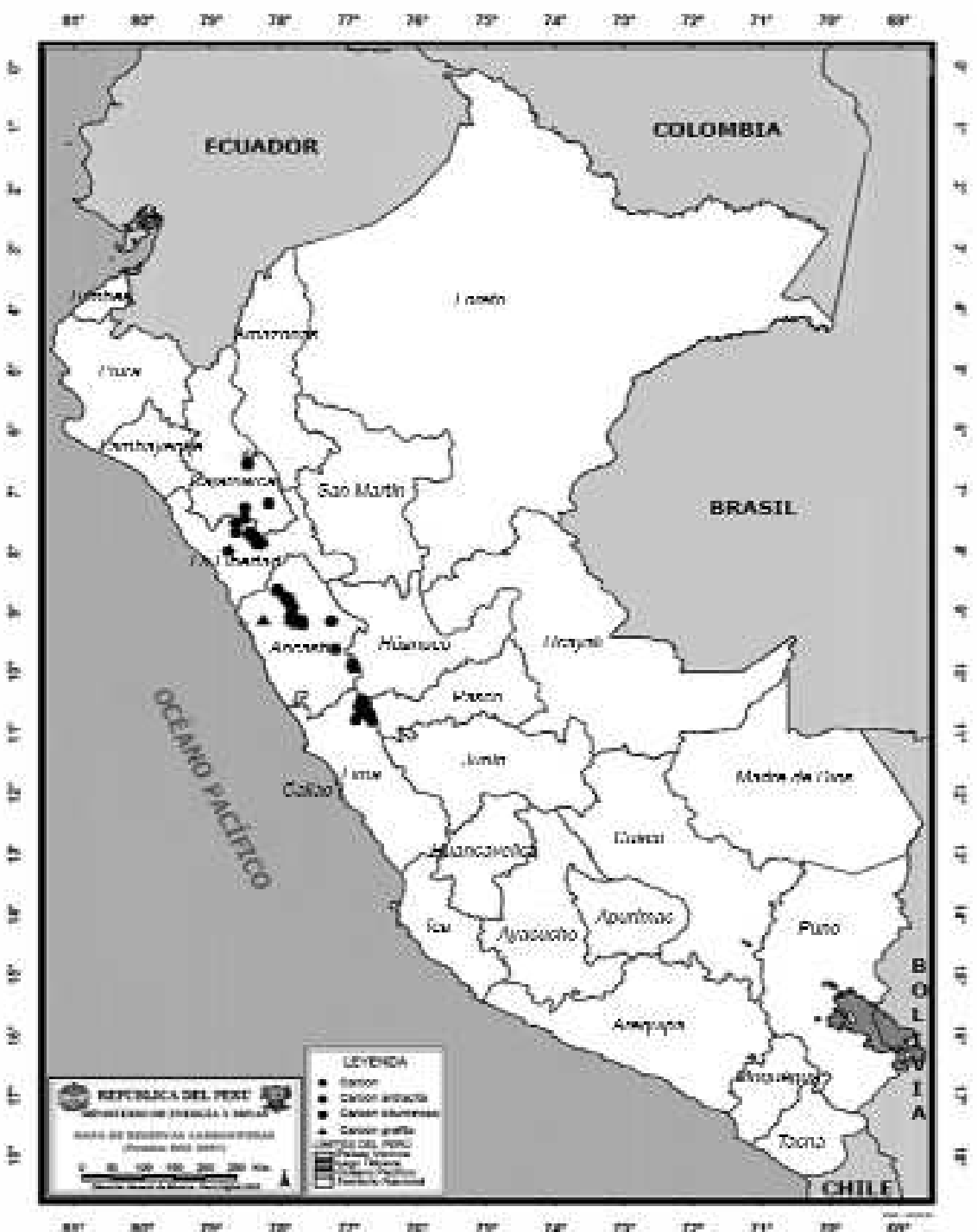

Fuente: Minem Perú (2001) 


\section{PROCESAMIENTO DE CARBÓN MINERAL}

En esta sección se presentan, en el nivel conceptual, un grupo de operaciones y procesos recomendables para el procesamiento de carbón mineral. Cabe anotar que estos procesos deberán desarrollarse dentro de una política general de buena práctica operativa, tecnología limpia, seguridad laboral, cuidado del ambiente y responsabilidad social con el entorno poblacional.

\subsection{Diagrama general de clasificación y lavado de carbón mineral}

El carbón mineral que se extrae del yacimiento minero debe pasar por una etapa de clasificación granulométrica, produciendo tres corrientes de material de $10 \mathrm{~mm}, 0,5 /-10 \mathrm{~mm}$ y $-0,5 \mathrm{~mm}$, que corresponden a las corrientes de grueso, mediano y fino, respectivamente. De la corriente de lavado de gruesos se obtienen los productos denominados gruesos lavados, mixtos de gruesos y estériles de gruesos.

De la corriente de lavado de medianos se obtienen los productos denominados medianos lavados, mixtos de medianos y estériles de medianos. El material fino pasa por una operación de flotación y filtración, dando como resultado dos productos: finos lavados y filtrados, y un efluente de flotación que se enviará a un sumidero, como se muestra en la figura 2 .

\subsection{Diagrama de lavado y clasificación de carbón}

En la figura 3 se presenta el esquema básico del lavado y clasificación de carbón mineral obtenido del yacimiento minero, con la finalidad de conseguir el carbón con diferente tamaño de partícula, clasificándolo en carbón grueso, carbón medio y carbón fino.

Esta clasificación se realiza en la tolva o depósito superior, donde se lleva a cabo la operación de flotación con agua del material carbonoso, para posteriormente desplazarlo por rebose a la rampa de filtros con mallas de diferente graduación. También por el fondo de depósito se descargan las impurezas que trae el carbón en bruto. 


\section{Figura 2}

Diagrama conceptual de clasificación de carbón mineral

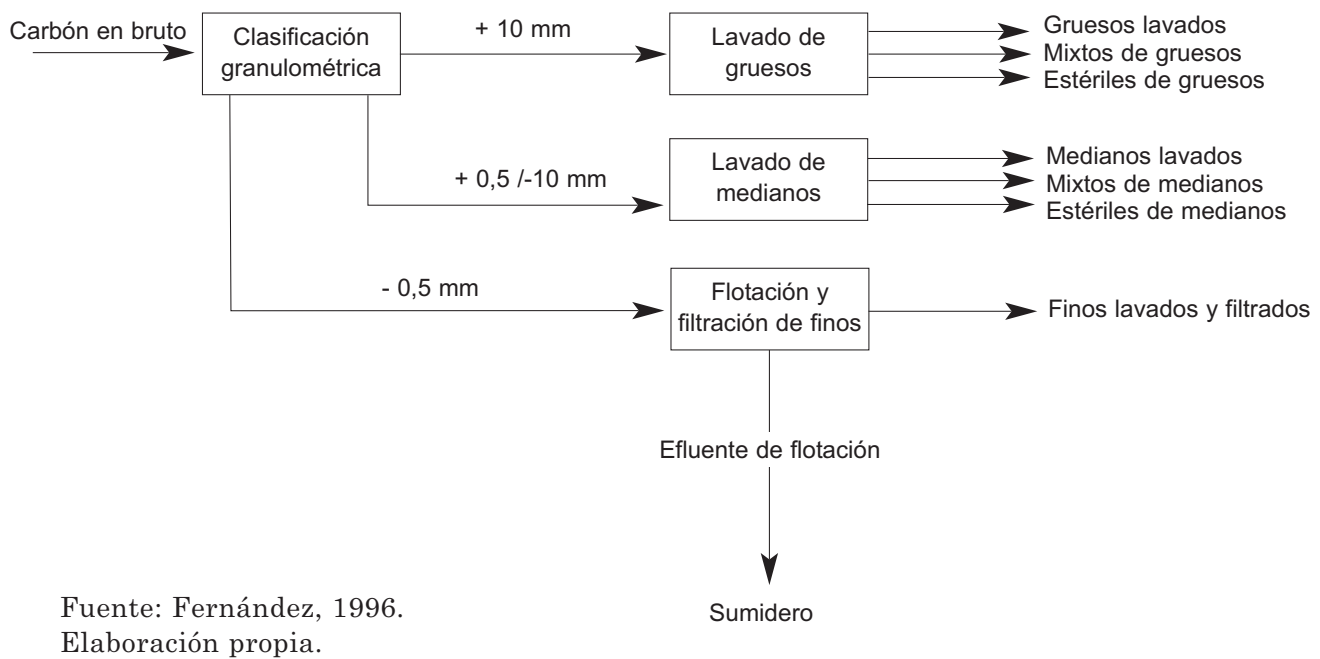

Figura 3

Unidad de lavado y clasificación de carbón

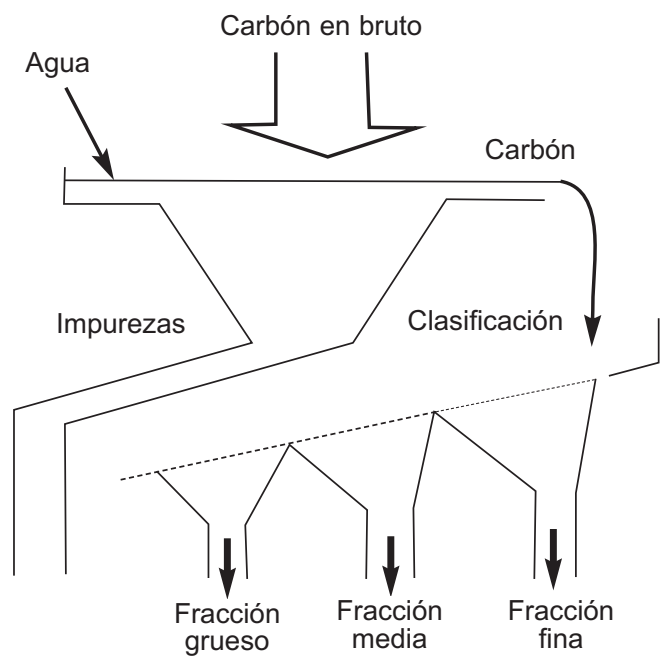

Fuente: Inusta, 2004.

Elaboración propia. 


\section{PROCESO DE INTEGRACIÓN DE GASIFICACIÓN CON CICLO COMBINADO (IGCC)}

En la figura 4 se expone el diagrama de uno de los sistemas más promisorios para generar potencia eléctrica y potencia térmica, empleando turbinas de gas de alta, media y baja presión, así como unidades de recuperación de calor, conectadas a un sistema de gasificación de carbón mediante el empleo de oxígeno puro proveniente de una planta de fraccionamiento de aire.

A este sistema se le conoce como proceso de Integración de Gasificación y Ciclo Combinado (IGCC). Como se emplea carbón como materia prima, se hace necesaria una etapa de eliminación de partículas inertes y otra de desulfuración. Es uno de los sistemas de mayor eficiencia general. El oxígeno es proporcionado por una unidad de fraccionamiento criogénico de aire. La modificación que se propone es emplear una tecnología de separación por membrana para obtener el oxígeno a partir del aire.

\section{PRODUCCIÓN DE HIDRÓGENO A PARTIR DE CARBÓN CON SEPARACIÓN POR ADSORCIÓN PRESURIZADA (PSA)}

De acuerdo con la figura 5, el carbón es alimentado a un gasificador para obtener una mezcla de monóxido de carbono e hidrógeno además de partículas inertes y $\mathrm{H}_{2} \mathrm{~S}$. El llamado gas de síntesis o syngas $(\mathrm{CO}+$ $\mathrm{H}_{2}$ ) pasa a un convertidor para conseguir mayor cantidad de hidrógeno. Luego, el $\mathrm{H}_{2} \mathrm{~S}$ es separado y usado para obtener ácido sulfúrico como subproducto. Se extrae el dióxido de carbono mediante un separador y el gas residual ingresa a una unidad PSA para obtener el hidrógeno con alto grado de pureza y el resto de gases sirve como combustible para una caldera. 
Perspectivas de procesamiento y uso del carbón mineral
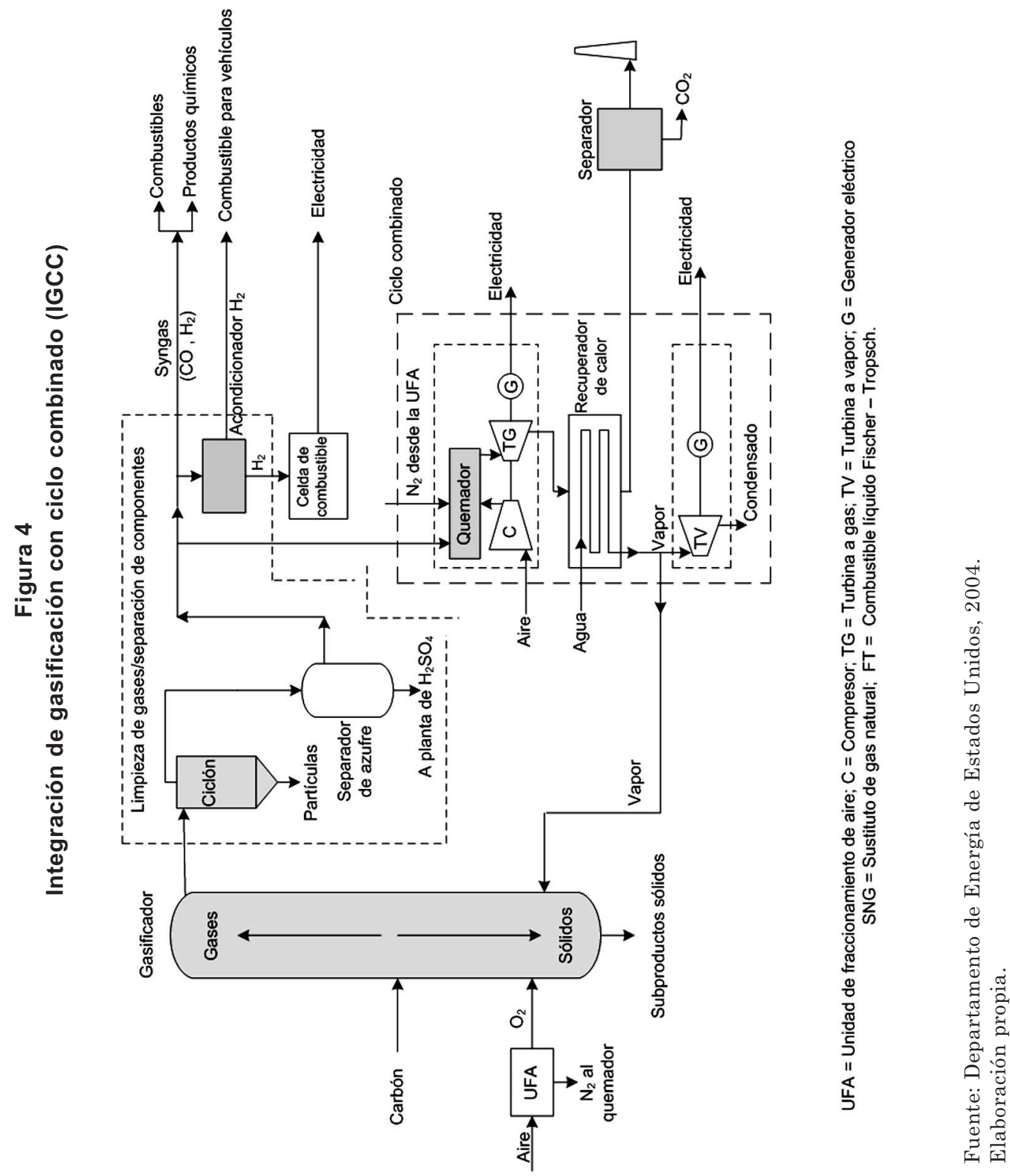
$\mathrm{CO}_{2}$
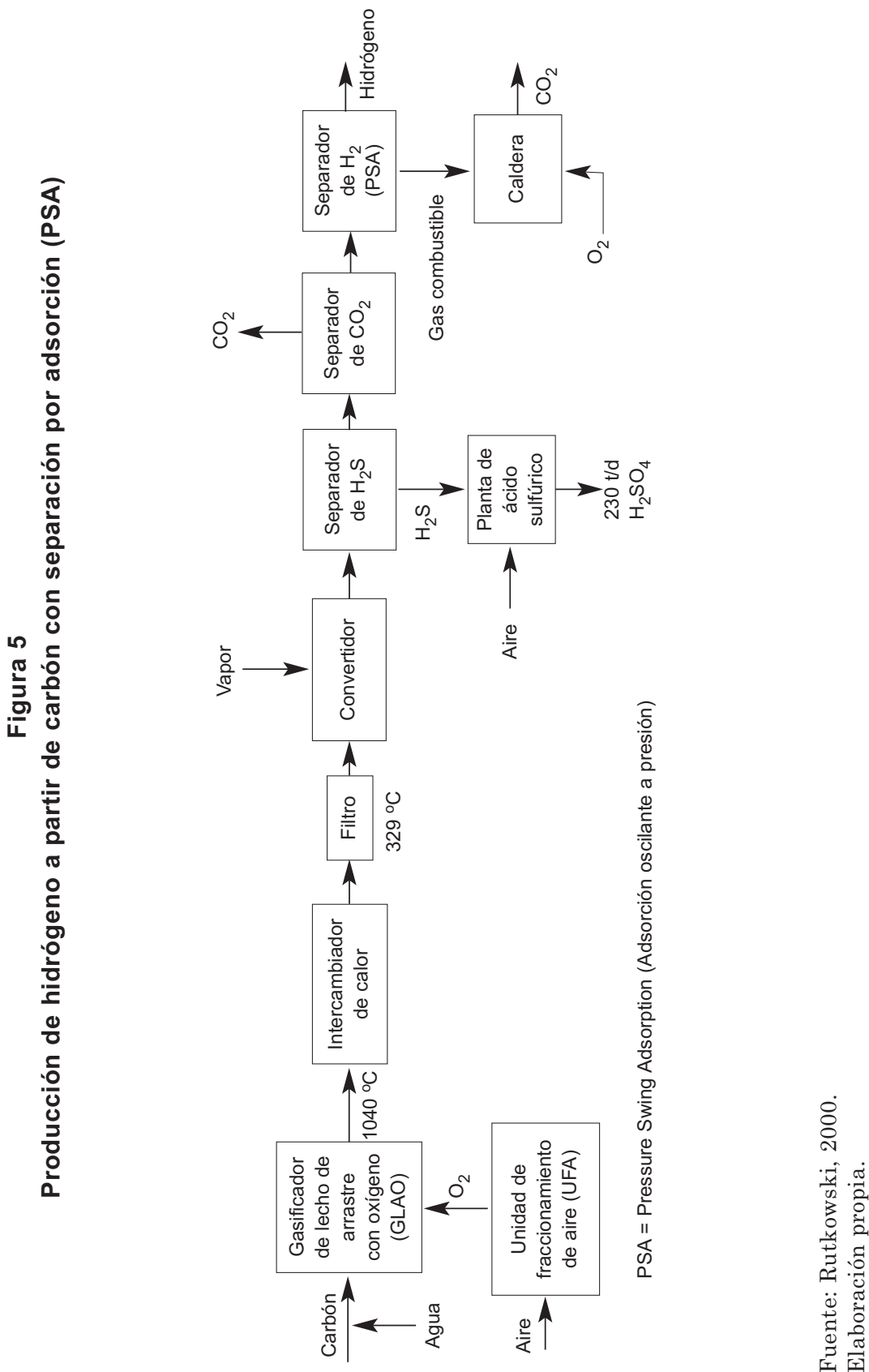


\section{PRODUCCIÓN DE HIDRÓGENO A PARTIR DE CARBÓN CON SEPARACIÓN POR MEMBRANA}

Este sistema productivo de hidrógeno es similar al proceso descrito anteriormente, pero emplea la separación del hidrógeno mediante membranas. El carbón es alimentado a un gasificador para obtener una mezcla de monóxido de carbono e hidrógeno, además de partículas inertes y $\mathrm{H}_{2} \mathrm{~S}$. El syngas $\left(\mathrm{CO}+\mathrm{H}_{2}\right.$ ) pasa a un convertidor para conseguir mayor cantidad de hidrógeno. Luego, el $\mathrm{H}_{2} \mathrm{~S}$ es separado y usado para obtener ácido sulfúrico como subproducto.

Se emplea una unidad de separación por membrana para obtener el hidrógeno con alto grado de pureza; una parte del gas residual $\left(\mathrm{CO}_{2}\right)$ ingresa a un quemador para generar gases que accionan una turbina de gas para la generación de electricidad, y la otra parte $\left(\mathrm{H}_{2}\right)$ pasa por un recuperador de calor para su compresión y enfriamiento, conforme se muestra en la figura 6 .

\subsection{Tecnología avanzada de combustión (PFBG-CFBC)}

Este sistema es un proceso híbrido que comprende gasificación y combustión. El carbón mineral ingresa con un sorbente a un gasificador de lecho fluido presurizado (PBBG). El gas producido es limpiado de azufre y de material sólido, y una vez limpio pasa a un quemador para producir gases que, a su vez, accionan una turbina de gas para generar electricidad.

El material sólido (coque inferior) se transfiere a un combustor de lecho fluido circulante (CFBC) que produce gases y vapor de agua mediante un recuperador de calor y un intercambiador de calor externo. Este vapor, a su vez, acciona una turbina de vapor para generar electricidad (figura 7). 
Mario Rojas Delgado

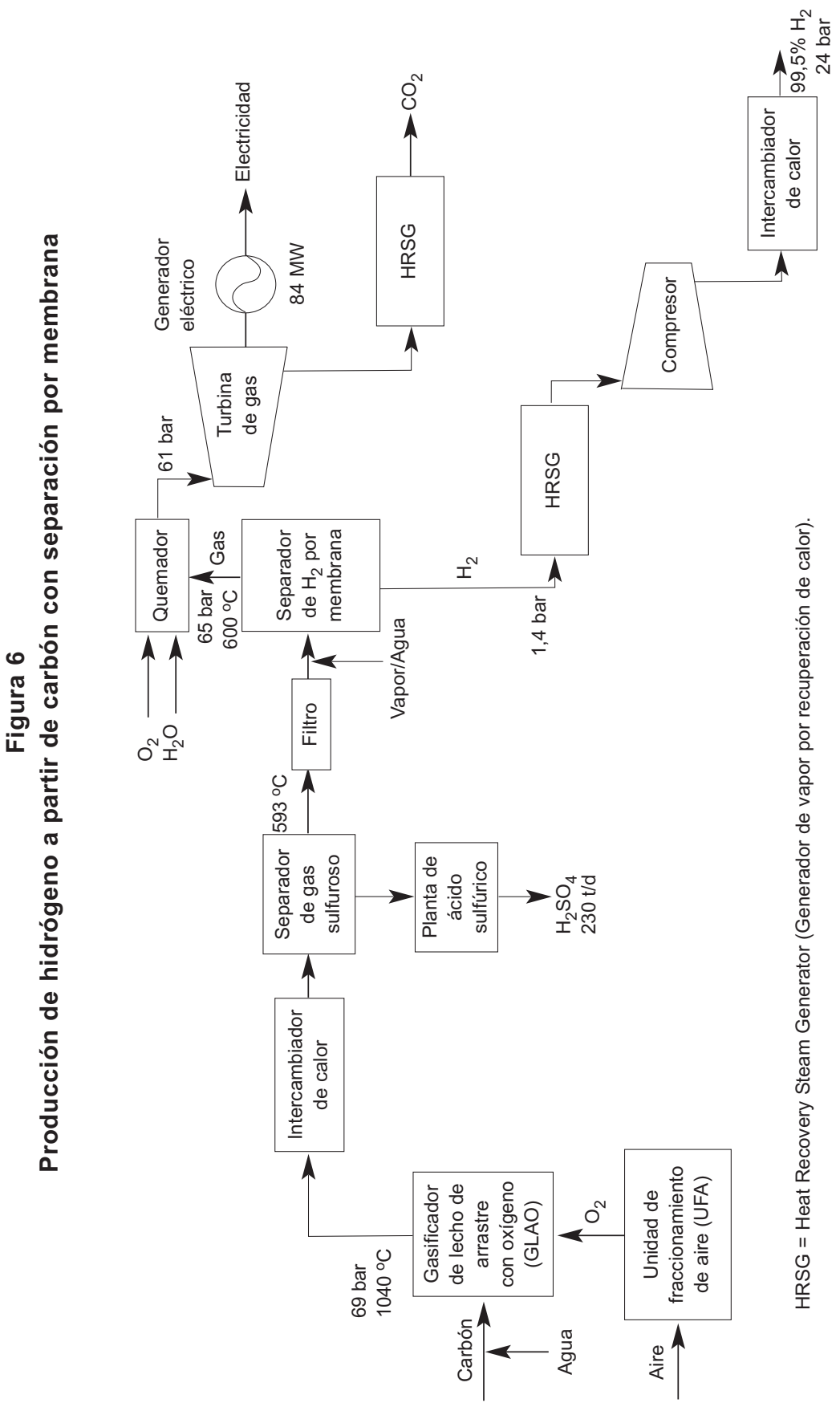

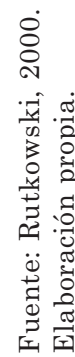


Figura 7

Tecnología avanzada de combustión (PFBG - CFBC)

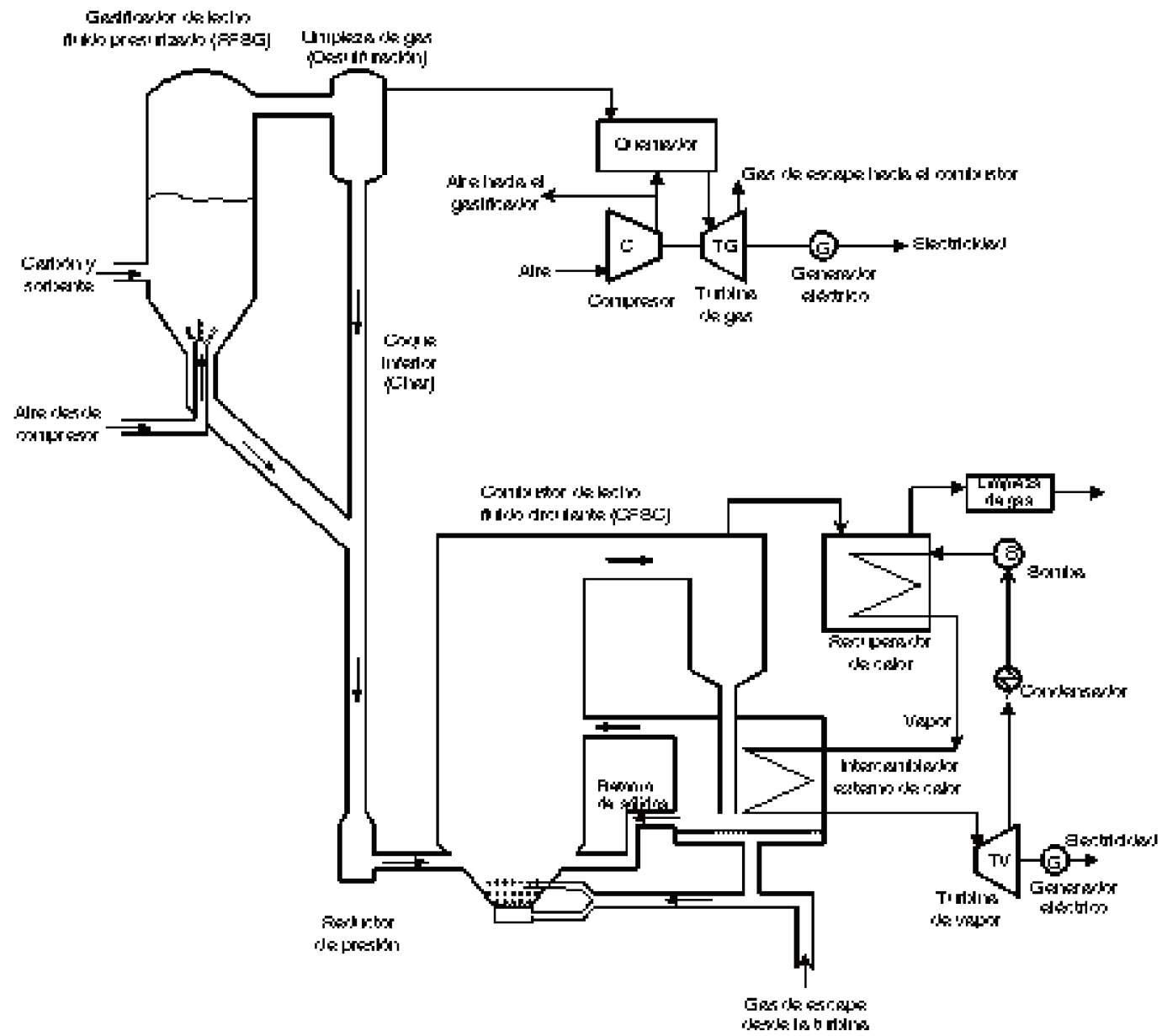

Fuente: Departamento de Energía de Estados Unidos, 2005.

Elaboración propia. 


\subsection{Combustibles tipo Fischer-Tropsch (FT)}

Para el desarrollo de los acápites siguientes nos basamos en Huffman (2005).

\subsubsection{Síntesis FT para obtener combustibles líquidos a partir de carbón}

a) Gasificación de carbón $\rightarrow$ syngas $\left(\mathrm{CO}+\mathrm{H}_{2}\right)$

b) La síntesis FT comprende hidrogenación y polimerización para producir combustibles líquidos, esencialmente libres de azufre y aromáticos:

1) $2 \mathrm{nCO}+\mathrm{nH}_{2} \rightarrow--\left[\mathrm{CH}_{2}\right]_{\mathrm{n}}-\mathrm{nCO}_{2} \quad$ (Catalizador: $\left.\mathrm{Fe}\right)$

2) $\mathrm{nCO}+2 \mathrm{nH}_{2} \rightarrow--\left[\mathrm{CH}_{2}\right]_{\mathrm{n}}-\cdot+\mathrm{nH}_{2} \mathrm{O}$ (Catalizador: $\mathrm{Co}$ )

\subsubsection{Desarrollo comercial de combustibles FT basados en carbón}

En Sudáfrica, SASOL produce 200.000 bpd con tecnología FT, y China ha anunciado planes para construir dos plantas FT de $80.000 \mathrm{bpd}$ cada una.

En Pennsylvania, Estados Unidos, se construirá una planta de 5.000 bpd con tecnología FT, teniendo como base el carbón antracita.

Bajo el concepto de poligeneración se usa el syngas para producir combustibles FT más limpios y electricidad mediante plantas IGCC (Integración de Gasificación con Ciclo Combinado).

Para el 2030, Estados Unidos planea reemplazar las importaciones de petróleo del Golfo Pérsico por plantas poligenerativas.

\subsubsection{Proceso de producción catalítica de metano (catalizador} propietario) a partir de syngas para la obtención del Sustituto de Gas Natural (SGN)

Este proceso se realiza mediante la metanación catalítica a partir del syngas $\left(\mathrm{CO}+\mathrm{H}_{2}\right)$ :

$$
\mathrm{CO}+3 \mathrm{H}_{2} \rightarrow \mathrm{CH}_{4}+\mathrm{H}_{2} \mathrm{O}
$$




\section{TIPOS Y USOS DEL CARBÓN MINERAL}

\subsection{Tipos de carbón}

El carbón, compuesto principalmente por carbono, hidrógeno, nitrógeno, oxígeno y azufre, es el resultado de procesos naturales de muy larga duración a partir de residuos vegetales.

En el cuadro 1 se muestra una tipología general, perteneciente a la American Society for Testing and Materials (ASTMD-388-777). Se indican cuatro tipos básicos, de acuerdo con la composición de los vegetales y las condiciones de presión y temperatura durante los procesos naturales para su transformación.

\section{Cuadro 1}

Clasificación general del carbón mineral

\begin{tabular}{lcccccc}
\hline Tipo & $\begin{array}{c}\text { Carbono } \\
\text { fijo } \\
(\%)\end{array}$ & $\begin{array}{c}\text { Materia } \\
\text { volátil } \\
(\%)\end{array}$ & $\begin{array}{c}\text { Contenido } \\
\text { humedad } \\
\mathbf{( \% )}\end{array}$ & $\begin{array}{c}\text { Poder calorífico } \\
\text { (BTU/lb) }\end{array}$ & $\begin{array}{c}\text { Poder calorífico } \\
\text { (MJ/kg) }\end{array}$ & $\begin{array}{c}\text { Poder calorífico } \\
\text { (kcal/kg) }\end{array}$ \\
& & & & & & \\
\hline Antracita & $86-98$ & 1 & $<15$ & $>14.000$ & $>32,6$ & $>7.780$ \\
Bituminoso (hulla) & $45-86$ & 32 & $15-20$ & $10.500-14.000$ & $24,5-32,6$ & $5.800-7.780$ \\
Subbituminoso & $35-45$ & 50 & $20-30$ & $7.800-10.500$ & $18,2-24,5$ & $4.300-7.780$ \\
Lignito y turba & $25-35$ & 96 & $>30$ & $4.000-7.800$ & $9,3-18,2$ & $2.200-4.300$ \\
\hline
\end{tabular}

Fuente: American Society for Testing and Materials (ASTMD-388-777); Minem, Colombia, UPME (2005).

La clasificación de ASTM (Estados Unidos) se puede comparar con las clasificaciones de UN-ECE (Naciones Unidas - Comisión Económica Europea) y de DIN (Alemania) que describen un mayor número de tipos de carbón (UN-ECE, ASTM, DIN, 2006).

\subsection{Usos del carbón}

- Antracita. También llamado carbón duro o carbón de piedra, posee un alto contenido de carbono ( $86 \%$ al $98 \%$ ), bajo contenido de material volátil y poder calorífico superior a $32,6 \mathrm{MJ} / \mathrm{kg}(14.000$ BTU/lb). Es utilizado como insumo en la industria siderúrgica, 
industria carboquímica y potencialmente en plantas carboeléctricas. Una perspectiva interesante es el uso de este tipo de carbón para la producción de hidrógeno, el cual puede ser aprovechado en celdas de combustible.

- Carbón bituminoso. Este tipo de carbón posee un menor contenido de carbono y menor poder calorífico que el carbón antracita. Por su uso se le conoce como carbón coquizable, es utilizado en procesos siderúrgicos, generación de vapor y producción de energía térmica.

- Carbón subbituminoso. Con menor poder calorífico que los carbones bituminosos, posee una composición en carbono entre $35 \%$ y $45 \%$ y un elevado contenido de material volátil. Es utilizado en la producción de energía eléctrica y energía térmica.

- Lignito y turba. Son carbonos con alta humedad y alto contenido de ceniza y de material volátil, lo cual hace que posean un bajo poder calorífico. Son usados para calefacción, energía eléctrica y generadores de vapor de agua (Minem, Colombia, UPME, 2005).

En el cuadro 2 se indican los usos actuales y potenciales para el carbón mineral en el Perú.

\section{Cuadro 2}

\section{Usos y productos del carbón mineral}

\begin{tabular}{lll}
\hline Industria / Servicio & Sector & Productos \\
\hline Siderúrgica & Minero-metalúrgico & Hierro esponja \\
Ladrillera & No metálicos & Ladrillos \\
Cementera & Industrial no metálicos & Cemento \\
Eléctrica & Energético & Electricidad \\
Hotelera & Servicios, poblacional & Briquetas \\
Carboquímica & Químico-industrial & Gas de síntesis \\
Agroindustria & Agrario & Fertilizantes \\
Energética & Combustibles & Hidrógeno \\
Química & Energético & Sustituto de gas natural \\
& & \\
\hline
\end{tabular}

Elaboración propia. 


\subsection{Estimado de costos de inversión y de procesamiento de carbón mineral en el nivel preliminar}

El cuadro 3 presenta cifras estimadas en el nivel preliminar, sujetas a replanteo, de costos de inversión y producción para las operaciones y los procesos relacionados con la industrialización del carbón mineral para los procesos tecnológicos citados en este artículo.

Cabe resaltar el abaratamiento de los costos de producción y de los costos de inversión que genera el empleo de la tecnología de membrana para la obtención de hidrógeno.

\section{Cuadro 3}

Capacidad, costo de inversión y costos de producción de plantas a carbón

\begin{tabular}{|c|c|c|c|}
\hline Planta & \multicolumn{2}{|c|}{ Capacidad operativa Costo de inversión } & Costo de producto \\
\hline $\begin{array}{l}\text { Extracción, lavado y } \\
\text { clasificación de carbón }\end{array}$ & $1.800 \mathrm{t} / \mathrm{mes}$ & $100 \mathrm{k}$ US\$ & $(60-80)$ Sol/t \\
\hline Planta eléctrica tipo PFBC & $100 \mathrm{MW}$ & $130 \mathrm{M}$ US\$ & 14 US $\$ / M W$ \\
\hline Planta poligenerativa IGCC & $100 \mathrm{MW}$ & 140 M US\$ & 14 US $\$ / M W$ \\
\hline Planta de $\mathrm{H}_{2}$ tipo membrana & $16.300 \mathrm{~kg} / \mathrm{h}$ & 363 M US\$ & 4,8 US\$/GJ \\
\hline Planta de $\mathrm{H}_{2}$ tipo PSA & $12.025 \mathrm{~kg} / \mathrm{h}$ & 375 M US\$ & 6,55 US\$/GJ \\
\hline
\end{tabular}

Fuentes: Consorcio Preeica, 2004; Rutkowski, 2000; U.S. DOE, 2004.

\section{CONCLUSIONES Y RECOMENDACIONES}

\subsection{Conclusiones}

La zona de mayor potencial en el Perú y recomendable para la carboindustria es la de Alto Chicama, en la región La Libertad.

Los factores de mayor riesgo en las operaciones de extracción de carbón mineral son los de explosión del gas metano, de inundación y de derrumbe en los túneles de extracción del carbón.

La actual capacidad de producción y tiempo de respuesta de los empresarios formales que extraen carbón en la zona del Alto Chicama, 
y que lo comercializan en Trujillo, permitiría cubrir la demanda proyectada de carbón antracita para los siguientes tres años.

La extracción de carbón informal es de tres a cinco veces mayor que la extracción formal.

La demanda de carbón antracítico en el Perú se puede expresar en función de los siguientes factores: producción de cemento, producción de ladrillo y volumen de producción de las industrias básicas del hierro y el acero, principales agentes consumidores de carbón mineral en sus procesos.

La integración de gasificación de carbón con ciclo combinado (IGCC) es el proceso más ventajoso, por ser poligenerativo y tener mayor eficiencia.

En la producción de hidrógeno con proceso de tecnología de membrana, resulta ser el más promisorio por ser el más eficiente en costos respecto al proceso de separación por criogenización.

El proceso Fischer-Tropsch (FT) para obtener combustibles líquidos a partir del carbón como sustituto de gas natural, es una alternativa que ya es empleada en diversos lugares del mundo, pero en el Perú aún no se le ha dado la debida importancia.

\subsection{Recomendaciones}

- Organizar el sector productivo de carbón convocando a empresarios, transportistas, comercializadores, autoridades y representantes en general de la región, para generar consenso y convicción sobre la conveniencia de operar asociadamente.

- Dar mayor valor agregado al carbón mineral en bruto para conseguir un mejor precio para el carbón procesado, mediante la aplicación de operaciones de lavado y clasificación granulométrica.

- Aplicar el proceso de gasificación integrada con ciclo combinado, empleando carbón mineral para obtener energía eléctrica, hidrógeno, ácido sulfúrico, combustibles gaseosos y energía térmica.

- Procesar el carbón mineral para producir un gas sustituto del gas natural mediante el proceso integrado de gasificación y ciclo combinado (IGCC) y el proceso Fischer-Tropsch. 
- Emplear el carbón mineral en procesos avanzados de combustión (PFBG- CFBC) para la producción de potencia eléctrica.

- Aplicar el carbón mineral procesado en las industrias química, metalúrgica, siderúrgica, así como en la de servicios.

- Establecer una cadena de carbón mineral que comprenda las etapas de extracción minera, pretratamiento de carbón, transporte, almacenamiento, tratamiento como fases intermedias entre el inicio y el fin de la cadena que comprende a los proveedores y clientes; además de la implementación de un sistema que permita administrar la información de las operaciones de cada uno de los agentes que intervienen en la cadena.

\section{BIBLIOGRAFÍA}

Consorcio Preeica. "Costos típicos para proyectos termoeléctricos" [en línea]. Tegucigalpa, 2004. <www.preeica.ca/el_consorcio.htm>.

Departamento de Energía de Estados Unidos. "Advanced combustion technologies" [en línea]. Washington, 2005. <http://www.fe. doe.gov/programs/powersystems/combustion/index.html>.

_. "Gasification Technology Research and Development" [en línea]. Washington, 2004-2005. <http://www.fe.doe.gov/programs/powersystems/ gasification/index.html>.

Dunin-Borkowsky, E. "Minerales industriales del Perú: Carbones" [en línea]. Ingemmet. Lima, 1996. <http://www.ingemmet. gob.pe/actividades/geol_econ_y_prosp_min/yacimientos_minerales_no_metalicos.htm.

Fernández, J. et al. "Depuración de vertidos a cauces públicos de los lavaderos de carbón de Hunosa"[en línea] Madrid, 1996 <aguas.igme.es/igme/publica/libro29/pdf/lib29/4a_po_2.pdf> [Consulta: 7 de enero del 206].

Huffman, G. "Coal in your tank: the ultra-clean transportation fuel of the future" [en línea]. Lexington, KY: Universidad de Kentucky, 2005. <www.nrcce.wvu.edu/docs/HuffmanWVUpresentation2005.ppt>.

Ingemmet. "Carbón en el Perú". [en línea]. Lima, 1996 <www.ingemmet. gob.pe>. 
Inusta, J. "El carbón" [en línea]. El Mundo. Madrid, 2004. <http:// aula.elmundo.es/aula/laminas/lamina1075976702.pdf>.

Minem Colombia, UPME. "El carbón en el mundo" [en línea]. Bogotá, 2005. <http://www.upme.gov.co/Docs/Cadena_carbon.pdf>.

Minem, Perú. "Reserva carbonífera de la región Ancash" [en línea]. Lima, 2003. <www.minem.gob.pe/archivos/dgm/mapas/sig/car_res/ maps/02_ancash.pdf>.

__ . "Reserva carbonífera de la región Cajamarca" [en línea]. Lima, 2003. $<$ www.minem.gob.pe/archivos/dgm/mapas/sig/car_res/maps/06_ cajamarca.pdf $>$.

__. "Reserva carbonífera de la región La Libertad" [en línea]. Lima, 2003. $<$ www.minem.gob.pe/archivos/dgm/mapas/sig/car_res/maps/13_la_ libertad.pdf $>$.

—_. "Reserva carbonífera de la región Lima" [en línea]. Lima, 2003. <www.minem.gob.pe/archivos/dgm/mapas/sig/car_res/maps/15_li ma.pdf $>$.

—. "Mapa de reservas carboníferas". [en línea]. <http://www.minem. gob.pe/archivos/dgm/mapas/sig/car_res/mask/01_peru_ca_2003.pdf>.

Rojas, M.; López, E. y S. Zaldívar. "Desarrollo de un modelo de cadena de suministro para el aprovechamiento industrial del carbón mineral en el Perú". Lima: Instituto de Investigación Científica de la Universidad de Lima, 2006.

Rutkowski, M. "Coal processing plants for hydrogen production with $\mathrm{CO}_{2}$ capture". [en línea]. Lima, 2001, Estados Unidos, 2000. $<\mathrm{http}$ ://www.netl.doe.gov/publications/proceedings/00/h2_00/ rutkowski.pdf>.

UN-ECE, ASTM, DIN. "Internationale klassifikation der kohle" [en línea]. Alemania, 2006. <http://www.kohlenstatistik.de/ftp/ Kohlearten.pdf>. 